\title{
Improved change detection with nearby hands
}

\author{
Philip Tseng $\cdot$ Bruce Bridgeman
}

Received: 13 September 2010/Accepted: 4 January 2011/Published online: 30 January 2011

(C) The Author(s) 2011. This article is published with open access at Springerlink.com

\begin{abstract}
Recent studies have suggested altered visual processing for objects that are near the hands. We present three experiments that test whether an observer's hands near the display facilitate change detection. While performing the task, observers placed both hands either near or away from the display. When their hands were near the display, change detection performance was more accurate and they held more items in visual short-term memory (experiment 1). Performance was equally improved for all regions across the entire display, suggesting a stronger attentional engagement over all visual stimuli regardless of their relative distances from the hands (experiment 2). Interestingly, when only one hand was placed near the display, we found no facilitation from the left hand and a weak facilitation from the right hand (experiment 3 ). Together, these data suggest that the right hand is the main source of facilitation, and both hands together produce a nonlinear boost in performance (superadditivity) that cannot be explained by either hand alone. In addition, the presence of the right hand biased observers to attend to the right hemifield first, resulting in a right-bias in change detection performance (experiments 2 and 3).
\end{abstract}

Keywords Change blindness - Body posture - Visual working memory $\cdot$ Visual short-term memory $\cdot$ VSTM

\footnotetext{
P. Tseng $(\bowtie) \cdot$ B. Bridgeman

Psychology Department, University of California,

Santa Cruz, SS2, Rm 277, 1156 High Street, Santa Cruz, CA 95064, USA

e-mail: tsengphilip@gmail.com

P. Tseng

National Central University, Jhongli, Taiwan
}

\section{Introduction}

The qualitative differences between the cognitive and sensorimotor visual systems have been well documented. Supporting evidence comes from how the two systems respond differently toward illusory visual stimuli, without arousing subjective awareness of such difference in healthy individuals (Milner and Goodale 1995; Vishton et al. 2007). Some patients with visual agnosopsia, a phenomenon that is termed action-blindsight, can also perform certain goal-directed behaviors above chance level despite their inability to "see" these objects (Danckert and Rossetti 2005; Perenin and Rossetti 1996). In our laboratory, we also tested this difference between the two visual systems with a shifted frame that biased participants' spatial reference, which resulted in static mislocalizations when measured with a verbal probe but not with a pointing action (Bridgeman et al. 1997, Tseng et al. 2010b). These differences originate from the different purposes that the two systems serve: the cognitive system supports visual analysis and identification, whereas the sensorimotor system supports real-time motor action (Bridgeman et al. 1997).

Recently, several studies have pushed this idea further and suggested that visual analysis of objects can be altered when one's own hands are near the display (Abrams et al. 2008; di Pellegrino and Frassinetti 2000; Reed et al. 2010; Reed et al. 2006). Using a covert attention paradigm (Posner et al. 1987), Reed et al. (2006) asked participants to simply place one hand near the computer display and found that reaction time (RT) to target onset shortened whenever their hands were present. These authors concluded that a spatial prioritization for attention was created when one's hand (or hands) is in proximity. Using visual search, inhibition of return, and attentional blink paradigms, Abrams et al. (2008) also reported slower 
shifts of attention when one's hands are near the display (see Thura et al. 2008, for similar effects in saccadic eye movements). Covering the hands did not attenuate the slowing effect of nearby hands (Abrams et al. 2008; Reed et al. 2006). Abrams and colleagues interpreted their findings by suggesting a mechanism that facilitates a detailed evaluation of objects for potential manipulation. Besides these studies using a RT measure, there has been only one study that reported an increased accuracy in spatial discrimination when hands are placed in proximity (Dufour and Touzalin 2008). The task involved an online discrimination of LEDs from different near- and far-hand positions; greater accuracy was observed only in the nearhand condition.

Based on these findings, the present study aims to provide a more stringent test of the nearby-hand facilitation with a change detection paradigm. Unlike previous tasks investigating the effects of nearby-hand positions, change detection is a complex task that requires use of visual short-term memory (VSTM). Successful change detection begins with attentional allocation, encoding, maintenance of object details, and ends with retrieval and comparison processes. Failure in any of these steps may render change detection unsuccessful. VSTM is especially important in this context because change detection requires one to constantly encode and retrieve information in and out of VSTM, thus making a suitable task to investigate processes related to working memory (Luck and Vogel 1997). Most important, change detection is also an attentionally demanding task for two reasons. First, one must be attending to a change (instead of merely looking) in order to be consciously aware of the change enough to report accurate detection (O'Regan et al. 2000). And second, the encoding, maintenance, and retrieval processes of information in and out of VSTM are all highly dependent on attention (Wheeler and Treisman 2002). Thus, any attentional prioritization of objects should directly benefit VSTM due to its impact on attention. Furthermore, if nearby hands should bias attention to orient toward any particular location (e.g., regions that are closer to the hands), objects in those locations should enjoy more coherent representations in VSTM, and thereby showing higher accuracy. This attentional bias, if any, should be visible by comparing change detection accuracy between regions. Therefore, the nature of the nearby-hand effect, whether positive or negative, can be observed from its influence on one's attention and VSTM via change detection performance. To this end, experiment 1 was designed with varying levels of difficulty (by manipulating the numbers of items to remember) to test whether findings on near-hand facilitations in RT can also be observed in accuracy. More important, can such facilitatory effects be observed in a change detection task that involves extensive high-level processes such as attention and memory? Experiment 2 probed the nature of the facilitatory effect and investigated which regions on the display were more likely to receive such attentional prioritization. In other words, is the attentional boost primarily benefitting regions that are closer to the hands or is the entire display equally prioritized? Experiment 3 tested the contribution from each hand alone.

\section{Experiment 1}

The present experiment used a change detection paradigm to test the hypothesis of enhanced visual processing near the hands. We used a classic change detection paradigm from Luck and Vogel (1997), which has often been used to determine the capacity of VSTM. The prediction here is straightforward: if nearby-hand positions really bring forth a detailed analysis of visual stimuli, then participants should be more sensitive to changes when they place their hands near the stimuli.

\section{Methods}

\section{Participants}

Twenty-two UCSC students participated in the study in fulfillment of course requirement ( 6 men, 16 women, mean age $=19$ ). Of the 22 participants, 20 were right-handed and 2 were left-handed. We included data from the 2 lefthanded participants in the analysis throughout this experiment, but taking them out did not change the results. All participants had normal or corrected-to-normal vision. All participants signed a written consent prior to the experimental session.

\section{Material}

The entire display extended $360 \times 360$ pixels in the center of the monitor. Distance to the screen was $48 \mathrm{~cm}$, and angular size of the display area was $14.4 \times 14.4 \mathrm{deg}$. The visual stimuli consisted of multiple-colored squares $(6 \times 6 \mathrm{~mm})$ on a gray background. These squares could appear in any random locations, but always kept a $4 \mathrm{~mm}$ minimum distance between each other vertically and horizontally. The available colors were black, white, blue, yellow, red, green, and violet. Colors were randomly assigned to each square.

Set sizes of 8 and 12 were used, which is similar to the classical change detection paradigm used to investigate VSTM capacity (Luck and Vogel 1997). We did not use set size 4 because our pilot data showed a ceiling effect in both 
hit rate and d'. In both set sizes, there was only one 'target' that would change color. Half of the trials did not contain any color change. Locations of the squares were always the same.

The entire display was organized as a $6 \times 6$ grid (boundaries not visible to the participants). Therefore, there were 36 possible locations in which a square could appear. Throughout the entire block, a target would appear in a location only once, resulting in 36 'change' trials and 36 'no-change' trials.

\section{Procedure}

Participants sat with their heads steadied by a chinrest. They were instructed to place both hands by the monitor frame (Figure 1). All participants responded with their dominant hand on a keypad, which was placed either on their lap (invisible to them; the hands-off condition) or mounted on the left or right side of the monitor (the handson condition). Half of the participants performed the two set 8 blocks first and half performed the set 12 blocks first. The orders of the hands-on and hands-off blocks were counter-balanced between the set sizes. Participants performed 20 practice trials prior to the formal sessions. The
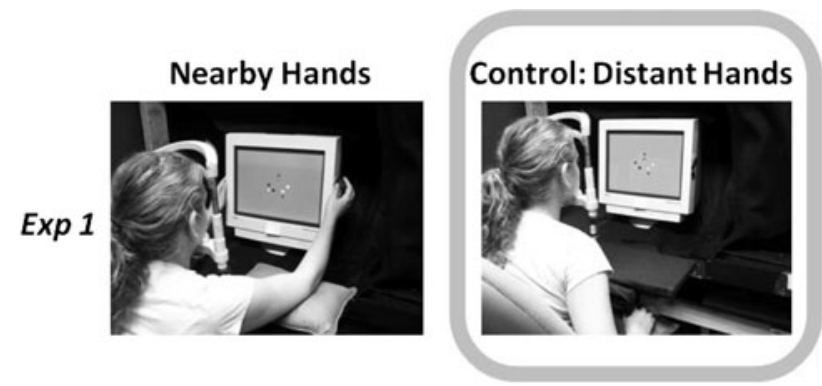

$\operatorname{Exp} 2$
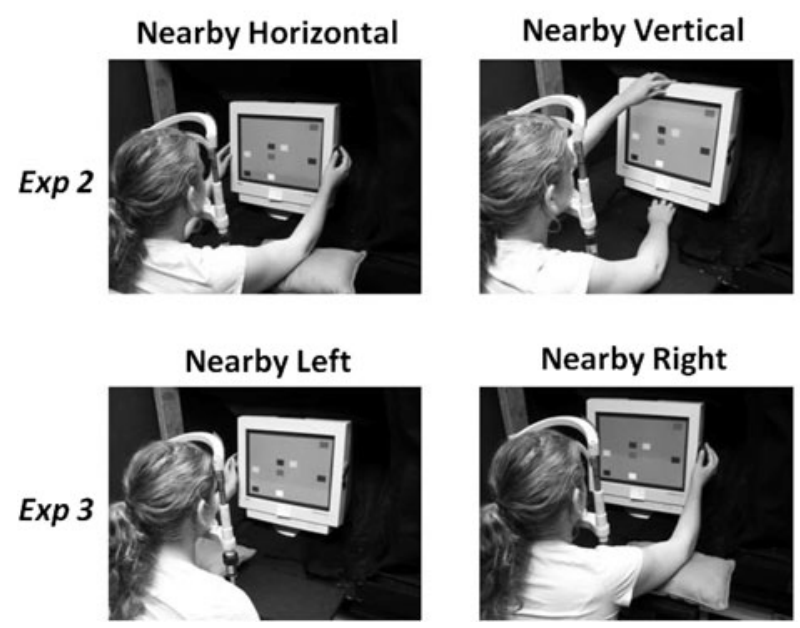

Fig. 1 Experiment setup and hand positions from all three experiments. The distant hand position in the control condition is the same across all experiments, but the stimulus display varies set size of the practice trials matched the set size of their first two formal blocks.

Each trial began with a 1000-ms fixation, followed by a 200-ms slide (A), a 900-ms interval, and ended with a 2200-ms slide (A').

We calculated each individual's d' scores from their hit and false alarm rates and submitted these d' scores to a $2 \times 2$ analysis of variance (ANOVA). The d' measure was used here because it accounts for one's response bias by incorporating the false alarm rate, thus representing one's true ability to detect changes. The two-way ANOVA consisted of independent variables of hand position and set size, with two levels under each factor (hands: on and off; set size: 8 and 12).

\section{Results}

We observed a significant main effect for hand position $(F=6.35, p=.02)$ and set size $(F=40.12, p<.01)$, but no interaction between them $(F=.12, p=.73)$. The novel finding here is the main effect of hand position regardless of task difficulty (see Figure 2a), indicating that participants were discriminating the items better when their hands were on the display, which led to better change detection.

To investigate whether such enhanced performance was related to an improved VSTM, we also calculated Cowan-Pashler's K (Cowan 2001; Pashler 1998) for each individual and submitted these scores to a $2 \times 2$ ANOVA. The Cowan-Pashler's formula assumes that each person can commit $k$ amount of integrated items in his or her VSTM at any given moment and specifies that the number $k$ can be approximated with the equation $k=\mathrm{N}$ (hits - false alarms). This formula takes false alarm rate into account and is often used to determine the capacity of VSTM in a given trial. The two-way ANOVA revealed similar findings (see Figure 2b): a significant main effect of hand position $(F=13.77, p=.001)$ and set size $(F=4.84, p=.04)$ with no interaction $(F=.07$, $p=.79)$. These results suggest that participants remembered more information when their hands were near the display.

We also analyzed RT data in a two-way ANOVA. The analysis revealed a marginally significant difference in set size $(F=3.57 ; p=.075)$ and no significant difference in hand positions $(F=.01 ; p=.925)$ or the interaction between set size and hands $(F=.096 ; p=.76)$.

\section{Discussion}

Previous studies have interpreted slower visual search rate near the hands as evidence of an enhanced visual analysis (Abrams et al. 2008; Davoli and Abrams 2009). The 


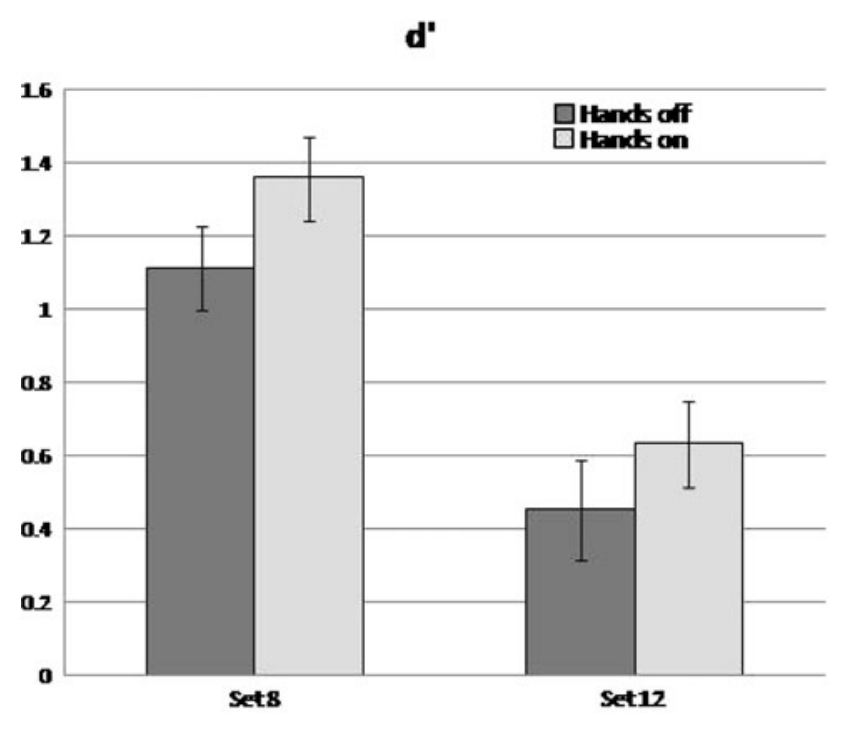

Cowan-Pashler's K

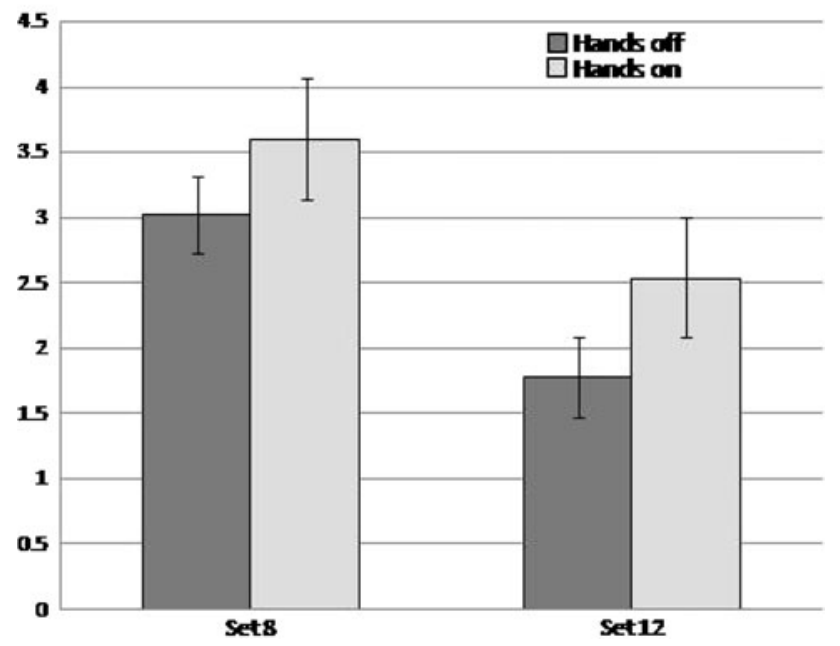

Fig. 2 Results from experiment 1. Both main effects of set size and hand positions are significant. Error bars represent standard errors of the mean

findings here suggest that the reported slower RT indeed reflected a facilitatory attentional process near the hands. In addition, the use of a change detection paradigm here revealed that the enhanced visual processing directly benefited participants' VSTM. On average, when participants' hands were positioned at the display, their memories held approximately 0.6 and 0.75 more items in sets 8 and 12 , respectively. This seems to imply an increase in VSTM capacity in the hands-on condition. However, although this interpretation is likely, it is not the most parsimonious explanation because it does not explain the nearby-hand effect in other paradigms that do not require an extensive use of VSTM. Another explanation would be that the nearby hands might have prioritized their adjacent areas for privileged attentional allocation (Reed et al. 2006), resulting in a better and deeper encoding of objection information into the VSTM. This attentional privilege would explain a memory in higher resolution and higher quantity. At any rate, the accuracy data here support the idea of an 'enhancement' of visual processing when one's hands are nearby.

The findings here also ruled out the possibility of a 'localized' facilitation of nearby-hand positions. Since the entire display was placed at the center of participants' vision (see Figure 1, top row), there were large margins (at least beyond grasp) between the hands and the actual stimuli. If the effect of nearby-hand positions were strictly localized to graspable locations, then no facilitation should have been observed in the present experiment. This still leaves two possible predictions undifferentiated, namely a 'graded' or 'uniform' pattern of facilitation from the hands. Specifically, the 'graded' pattern refers to a continuum of facilitation strength that increases in magnitude as the stimulus gets closer to the hand. This would predict stronger facilitation on the side of the display (near the hands) and weaker facilitation at the center. On the contrary, a 'uniform' pattern implies that any stimulus between the hands would get equally facilitated regardless of its distance from the hands. Thus, in experiment 2 , we attempt to investigate whether all items were equally attended or items positioned closer to the hands would get more facilitation.

\section{Experiment 2}

Experiment 1 showed that nearby hands can facilitate change detection performance regardless of task difficulty. Given this finding, the next question we ask is whether the nature of the facilitatory effect is graded or uniform. That is, does the magnitude of such facilitation increase as the stimuli are positioned closer to the hands? Or is it uniformly distributed, enhancing visual processing of nearby objects by the same magnitude regardless of their relative distances. To answer this question, we made a few modifications to our experimental setup. First, the visual display stretched to cover the entire monitor so that all stimuli can be positioned anywhere from the center of the monitor to where the hands were placed. Second, in addition to the left and right hands-on (hands-horizontal) positions, we also added a top-and-bottom (hands-vertical) condition. Therefore, if the facilitation was of a graded nature, we should observe an improvement in performance that, from the center of the display, spreads outward horizontally in the hands-horizontal condition and vertically in the handsvertical condition. 
Methods

\section{Participants}

Twenty-two UCSC students participated in the study in fulfillment of course requirement (9 men, 13 women, mean age $=19$ ). All had normal or corrected-to-normal vision. All participants signed a written consent prior to the experimental session. One participant's data was excluded from analysis due to several unintended interruptions during the experimental session. Data from the remaining 21 participants were used for the analyses described later. Of these participants, one reported to be left-handed, but removing this participant's data does not change the results that we report later.

\section{Material}

The hardware setup and the visual stimuli were identical to those used in experiment 1 , with the exception of display size. We increased the size of all the visual stimuli and the gaps between them so that the display covered the entire display area of the monitor $(41 \times 31$ degrees $)$. To accommodate the rectangular display area of the monitor, all colored squares from experiment 1 were changed to rectangles (16 $\mathrm{mm}$ wide and $13 \mathrm{~mm}$ tall). These rectangles were at least separated by a vertical distance of $10 \mathrm{~mm}$ and a horizontal distance of $24 \mathrm{~mm}$.

Besides the original hands-off and hands-on conditions, we added another hands-on condition that required participants to put their hands on the top and bottom of the computer monitor. Order of the three blocks (hands-off, hand-horizontal, hand-vertical) within each set size was pseudo-randomized between participants.

\section{Procedure}

The experimental procedure was identical to experiment 1 except for two changes. First, we increased slide A duration to $200 \mathrm{~ms}$ to allow participants to cover the bigger display. Second, since our pilot subjects experienced fatigue during the new hands-vertical block, we added an inter-trial interval that lasted indefinitely until a key was pressed by the participant. During this time, participants could either rest for as long as they wanted or explicitly request to switch hands between top and bottom to continue. Otherwise, all participants were asked by the experimenter to switch top and bottom hands halfway (between the 36th and 37th trial) through the vertical-hands block (72 trials total). Out of the 21 participants, 7 did request to switch top and bottom hands before the halfway point and continued to switch hands once or twice more at their own pace until the end of the block. Others only switched hands once when requested by the experimenter halfway through the block. Note that when they did switch their hand positions, the keypad was also relocated to where their dominant hand was. Both hands were facing down in the hands-vertical condition for all participants.

\section{Analysis}

The purpose of this study is to see whether the nearby hands would facilitate all regions on the display in a graded or uniform manner. Since false alarm rates cannot be broken down by regions, we used hit rates instead of d' for the regional gain analysis.

The entire display was composed of 6 columns and 6 rows, resulting in 36 possible locations of change (see experiment 1 for complete details). The analysis of interest here is to compare performances across different columns and rows while keeping hand positions in mind. If the hand facilitatory effect was graded, the columns (or rows in the hands-vertical condition) on the side should enjoy higher hit rates than those columns toward the center. To increase statistical power, we condensed data from the 6 columns and rows into 3 thicker columns and rows. Therefore, each column and row now contained twice the number of trials (e.g., in the horizontal condition, the left column contains trials from the two left-most columns, and the same for middle and right columns). Given the limited number of trials from each column and row, this aggregation made the statistical comparison more reasonable by keeping the number of factor levels low (the one-way ANOVA is now comparing between 3 columns or 3 rows instead of 6 ) and adding more trials under each level. For example, in the hands-horizontal condition, the left and right columns are close to the hands, whereas the middle one is not. Therefore, if the hand facilitatory effect was graded, the three condensed columns should yield higher hit rates on the side (left and right) than in the middle. Similar prediction also applies to the top and bottom rows in the hands-vertical condition.

Before submitting these nine hit rates into an ANOVA, another data transformation was necessary to account for participants' center bias. Naturally, when performing a change detection task, stimuli in the center are usually seen first, thus giving these items higher hit rates than the rest. Therefore, the effects of nearby-hand positions may have been masked, or canceled out, due to these two parallel advantages that are taking place. To control for this issue, we used the 9 hit rates from the hands-off condition to serve as a baseline control. In other words, the hands-off condition represents a scenario where the hands-on facilitation is absent, but participants' center-biased attention is present. We took the hit rates from each hands-on condition and divided them by the baseline hit rates of their 
corresponding cells from the hands-off condition. These ratios, then, represent facilitations from the nearby hands in each particular cell when attentional bias is controlled. These hit ratios were then aggregated and submitted to a one-way repeated-measures ANOVA. We also calculated a d' measure for each participant and submitted these data to a repeated-measures two-way ANOVA with three levels under hand positions (hands-off, hands-horizontal, handsvertical) and two levels under set size (8 and 12).

Results

The $3 \times 2$ ANOVA on d' revealed a significant main effect of hand position $(F=7.19, \quad p=.002)$ and set size $(F=40.63, p<.001)$ with no interaction $(F=.66$, $p=.52$ ). To compare the effect of hand positions under each set size, we performed Fisher's LSD for pairwise comparisons between hands-off, horizontal, and vertical hand positions under set size 8 and 12 . Under set 8 , averaged d' from the hands-off condition $(\mathrm{M}=.86, \mathrm{SD}=.45)$ was significantly lower than d' from the horizontal-hands $(\mathrm{M}=1.06$, $\mathrm{SD}=.53)$ and vertical-hands $(\mathrm{M}=1.07, \mathrm{SD}=.53)$ conditions. These two hands-on conditions did not significantly differ from each other. Under size 12, the same trend was also apparent, but only the difference between hands-off $(\mathrm{M}=.36, \mathrm{SD}=.46)$ and vertical-hands conditions was statistically significant $(\mathrm{M}=.63, \mathrm{SD}=.32)$. These results replicated our observations from experiment 1 .

Two-way ANOVA on Cowan-Pashler's $\mathrm{K}$ also indicated a significant main effect for hand positions $(F=6.87, p=.003)$ and set size $(F=11.48, p=.003)$, with no interaction between them $(F=1.01, p=.37)$. Pairwise comparisons revealed that means from the vertical-hands condition (set $8: \mathrm{M}=2.75, \mathrm{SD}=1.33$; set 12 : $\mathrm{M}=2.38, \mathrm{SD}=1.12$ ) were higher than the hands-off condition (set $8: \mathrm{M}=2.17$, $\mathrm{SD}=1.13$; set $12: \mathrm{M}=1.44$, $\mathrm{SD}=1.46$ ) in both set 8 and 12 . The difference between the horizontal-hands (set $8: \mathrm{M}=2.61, \mathrm{SD}=1.22$; set 12 : $\mathrm{M}=1.61, \mathrm{SD}=1.13)$ and the hands-off condition was only marginally significant under set $8(p=.09)$ and was not significant under set $12(p=.66)$. Together, these results from d' and Cown-Pashler's $\mathrm{K}$ indicate a lessened effect of nearby-hand positions with increased task difficulty (set 12). This was possibly caused by the increased display size, resulting in less time to properly encode the whole visual scene. However, the significant main effect of nearby-hand position was still present, suggesting a robust prioritization of the visual space near the hands. Notably, the facilitatory effects from the vertical hand positions seemed even stronger than the horizontal and were resistant to increased task difficulty (set 12; see Figure 3). This effect was also coupled with a slower RT $(\mathrm{M}=851.31 \mathrm{~ms})$ than the hands-off $(\mathrm{M}=786.71 \mathrm{~ms})$
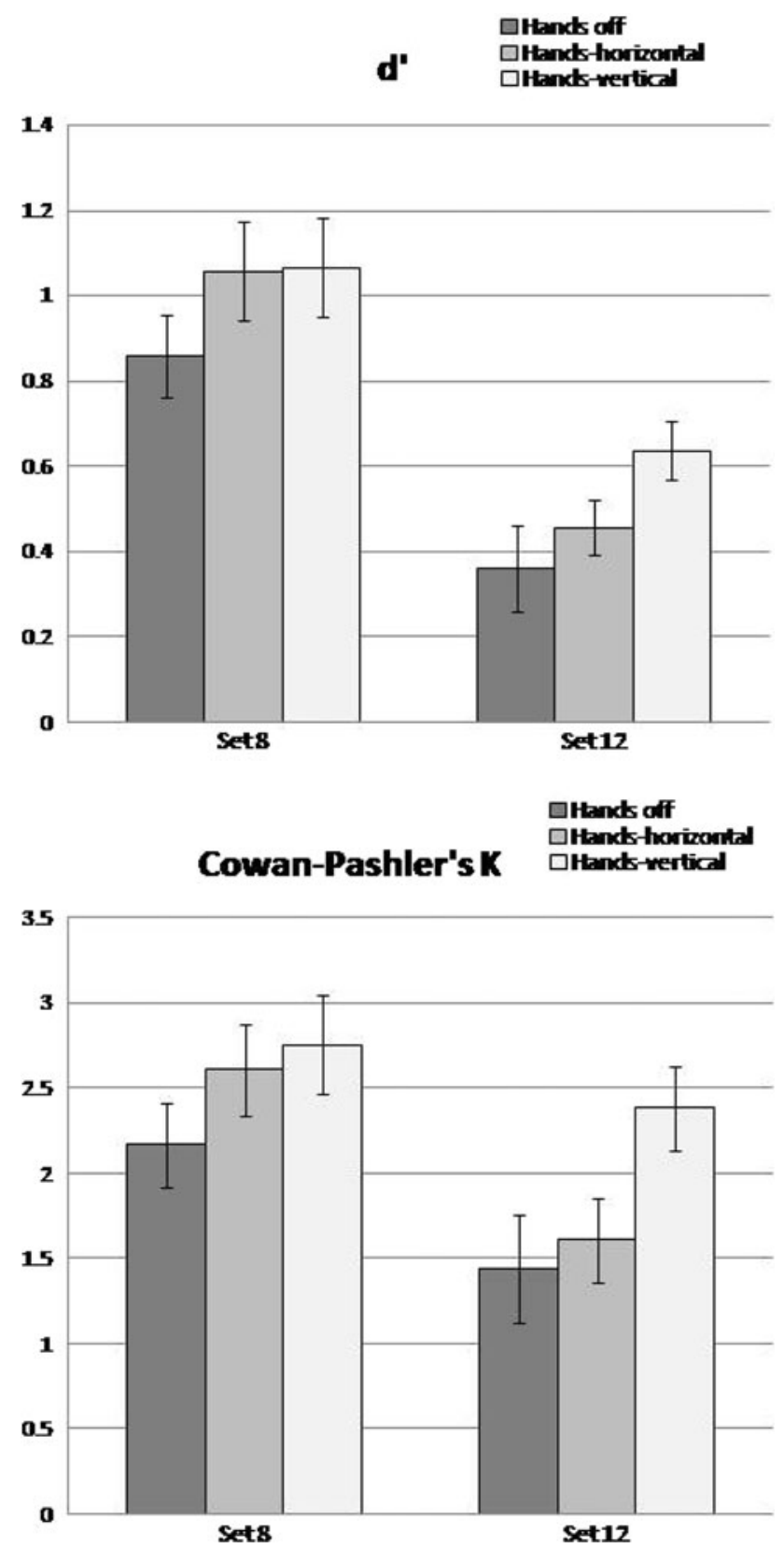

Fig. 3 Results from experiment 2. Both main effects of set size and hand positions are significant. Error bars represent standard errors of the mean

and the $(\mathrm{M}=763.18 \mathrm{~ms})$ horizontal-hands condition in a repeated-measures two-way ANOVA (set size: $F=.44$, $p=.51$; hand: $F=9.185, p=.001$; set $\times$ hand: $F=.05$, $p=.95)$. This effect in accuracy and RT was true across both set sizes and both measures.

\section{Regional analysis}

This study was designed to see whether the nearby-hand facilitation was graded or uniformly distributed. As previously described, we computed the hit ratio of each 
column and row from both hands-on conditions, aggregated the 6 columns and rows into 3 to increase statistical power, and submitted them into separate one-way ANOVAs. The ANOVAs revealed no significant overall differences in all four conditions (set 8 vertical: $F=1.34$, $p=.29$; set 8 horizontal: $F=1.02, p=.38$; set 12 vertical: $F=1.12, \quad p=.89$; set 12 horizontal: $F=1.96$, $p=.17$ ). Regrouping the columns and rows to compare the left-most and right-most (or top and bottom) single column with the middle 4 columns also did not yield any significant differences in ANOVA (set 8 vertical: $F=.24, p=.79$; set 8 horizontal: $F=.785, \quad p=.47$; set 12 vertical: $F=1.25, p=.31$; set 12 horizontal: $F=1.73, p=.2$ ). However, when we group the left and right 3 columns together, thus comparing left and right hemifields (or top and bottom), there was a significant difference under set size 12 horizontal condition (left: $100 \%$, right: $128 \%$, set 12 horizontal: $F=7.25, p=.01$; set 8 vertical: $F=1.89$, $p=.18$; set 8 horizontal: $F=.58, p=.46$; set 12 vertical: $F=.7, \quad p=.42$ ). This right-bias under high task difficulty is quite different from a graded facilitation, which we discuss in more detail later. Together, the data here suggest that even with the existing hemifield difference, the facilitation from the hands is uniformly distributed within each hemifield such that stimuli that are closer to the hands received equal amount of facilitation as stimuli that are further away.

\section{Discussion}

In this experiment, we observed similar nearby-hand facilitation as in experiment 1 with a bigger display size. We computed the gains in hit rate for each of the 6 columns and rows to see how they were distributed across the display. These gain ratios were computed by dividing each column and row from the hands-on condition (horizontal and vertical) by its corresponding column and row from the hands-off condition, thus accounting for participants' tendency to look at the center region (i.e., areas around the fixation point). Most of the regional gains were quite stable and evenly distributed (Figure 4). None of the columns and rows was significantly different from each other in any condition, suggesting a uniform improvement in change detection performance near the hands. Therefore, the visual system seems to perform an equally extensive analysis for all items on the display. This suggests that it is not the hands per se that are attracting attention, which would predict a localized facilitation near the hands. Rather, the nearby hands create a stronger attentional engagement (Abrams et al. 2008; Reed et al. 2006) with the display as a
Fig. 4 Regional gains in hit rates from experiment 2 . These gains were obtained by subtracting the each grid in the hands-off condition from their corresponding grid in the two hands-on conditions. Error bars represent standard errors of the mean
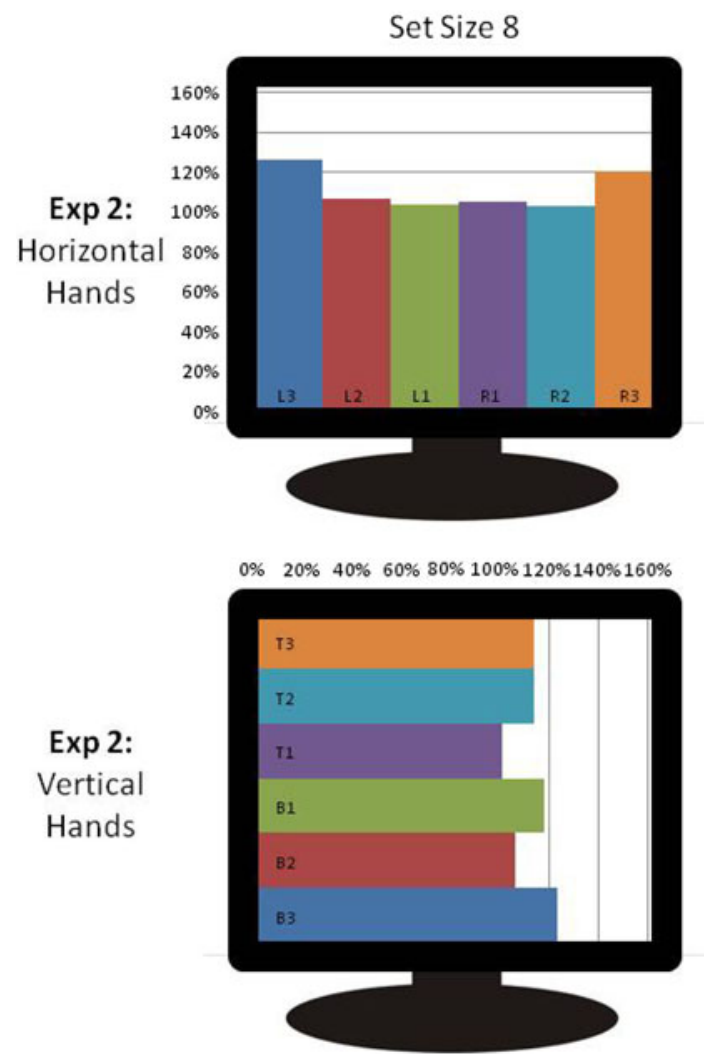
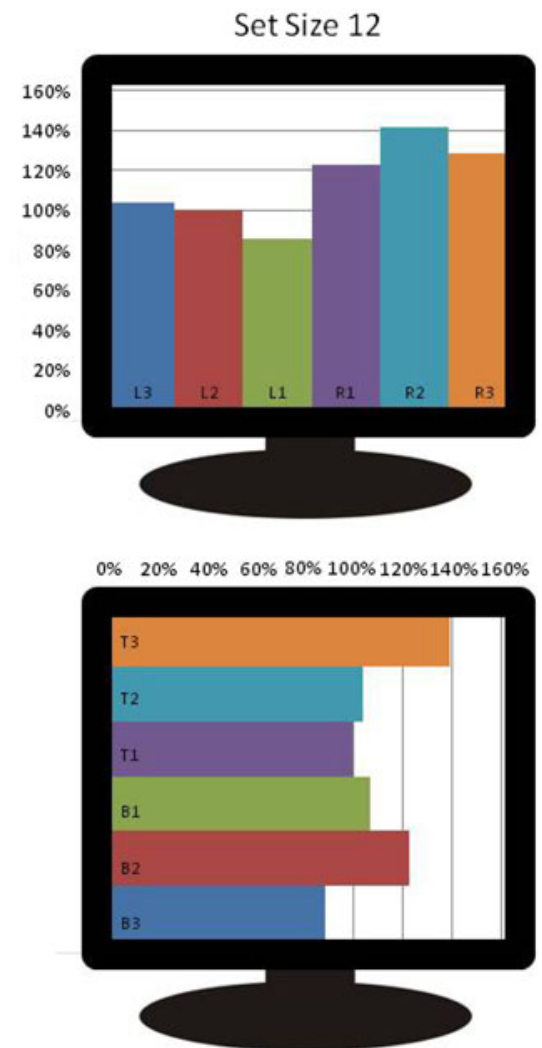
whole. However, it is important to note that the present findings do not entirely rule out the graded distribution perspective. That is, despite the flat regional gains, it is possible that the facilitations have a Gaussian distribution that peaks near the hands. This would create two peak facilitations on the left and right side, which would mask the graded facilitation because the two distributions are overlapped. To address this, we conducted experiment 3 with a single-hand design to get a complete picture of the facilitation distribution from each hand.

\section{Robust vertical-hands effect}

We observed a stronger facilitation when hands were placed vertically (Figure 3). This effect was robust and remained significantly better than the other conditions when task was difficult (set 12). This is a surprising finding as we did not expect the vertical effect to be any stronger than the horizontal. Although participants were given the opportunity to take extended rest on a trial-by-trial basis under this condition, they rarely did so (at most 2-3 times within the block). This implies that there was not sufficient frequency of utilized resting periods to affect the results in the magnitude we have observed here. One possible explanation is that the vertical hand positions required much more effort to maintain. As a result, the greater physical effort created a greater salience of the motor posture. However, Davoli and Abrams (2009) have reported a replication of the Abrams et al. (2008) study with imagined nearby-hand postures (relative to imagined hands placed behind participants' back). Therefore, the physical effort associated with the hand placement is unlikely to be the only key here. Perhaps, a simpler explanation is that by giving our participants a self-paced pausing period between the trials, the chances of missing an image from the following trial (picture A or A') due to blinks became less likely.

\section{The 'right' bias from set size 12}

Results from this study suggest a uniformly enhanced detection of objects near the hands. This can be explained by an attentional prioritization of the visual space within arms' reach (Reed et al. 2006). Presumably, objects near the hands represent items that are more likely to provide action possibilities. Therefore, it is not surprising that these objects should receive prioritized allocation of attention. This logic, however, hits a stall point when the prioritized region gets too big or if the number of stimuli within one area gets too large. For example, when the size of the prioritized region gets beyond the coverage of one single saccadic eye movement, is there a prioritized location among all the prioritized regions? Similarly, if too many stimuli are competing for attentional resources within one given area, it is plausible that certain areas are prioritized first. These scenarios are possible and are likely the case in our set size 12 condition because there were simply too many stimuli to encode within a short amount of time. To answer this question, our data seems to suggest a yes for horizontal hand positions and a no for vertical hand positions. In the set size 12 condition, whenever hands were placed horizontally, the right side always enjoyed better detection than the center and the left side (see Figure 4 upper right graph). This is somewhat different from a graded facilitation, because change detection of the left side is no better than the center. Rather, there is a strong bias toward the right side and the right side only. The vertical-hands condition also did not show a similar distinction between the top and bottom panel despite its greater overall facilitation than the horizontal-hands condition. This dissociation between the two hands-on conditions also ensures that the right-side bias was not due to an insufficient statistical power to uncover facilitations on the left side because the vertical-hands condition had a stronger effect yet showed no top or bottom bias. This bias to the right may be related to handedness since all but one of our participants were right-handed in experiment 2. This brings out another interesting question: When both hands are around the display, is there a possible competition for attentional prioritization between the two hemifields? If this is the case, our results here suggest a dominance of the right visual field in most of our participants, which is consistent with a recent investigation on the nearby-hand effect that reports facilitated RT only from the right hand (Lloyd et al. 2010).

Why did we not observe a directional bias when hands were vertically positioned? The simplest explanation is that there is no directional bias in the vertical space. Another possibility is that the right bias also exists in vertical hand positions, but such effect was averaged out when our participants switched hands between top and bottom.

\section{Experiment 3}

The present experiment is designed to address the possibility of an overlapping facilitation from both hands. As mentioned earlier, it is possible that the left and right hand provides a peak boost of performance on the left and right side, respectively, and gradually decays in a graded fashion. This overlapping distribution from the left and right would mask the graded nature, as observed in experiment 2 , and would require a single-hand condition to disentangle. In addition, besides the graded-or-uniform question, another goal of the present study is to dissociate the individual contribution from each hand. For example, the left 
and right hand do not necessarily need to contribute equally.

Methods

\section{Participants}

Forty-six UCSC students were recruited in fulfillment of course requirement (21 men, 25 women, mean age $=18$ ). All had normal or corrected-to-normal vision. All participants gave written consent prior to the experimental session. All participants were right-handed. One participant decided to opt out of the experiment; data from the remaining 45 individuals were used for the analysis.

\section{Material and procedure}

The materials and procedures were identical to experiment 2 with two exception regarding hand positions. First, there was no vertical-hands condition. Second, the horizontalhands condition was broken down into a left-only and a right-only condition. Together, there were six blocks in the formal experiment: two set sizes (size 8 and 12) with three hand positions (left-only, right-only, hands-off) under each set size.

\section{Results}

A $3 \times 2$ within-subjects ANOVA was conducted on participants' d' scores to compare the effect of single hand on change detection in left-only, right-only, and hand-off conditions (Fig. 5). There was a significant effect of set size $(F=109.05, \quad p=.71)$, no effect of single hand $(F=1.76, p=.19)$, and a significant interaction between them $(F=3.2, \quad p=.05)$. Separate one-way ANOVA under each set size revealed that the significant interaction was driven by a significant single-hand effect that was present under size $8(F=4.92, p=.01)$ but not size 12 $(F=.8, p=.46)$. Post hoc LSD comparisons under set size 8 showed that the right-hand condition $($ mean $=1.11)$ had significantly better performance than the lefthand (mean $=.97 ; \quad p=.04$ ) and hand-off conditions (mean $=.94 ; p<.01)$. There was no difference between the left-hand and hand-off performances. Overall, this is a somewhat surprising finding compared to what we have previously found because we did not observe a nearbyhand effect under set size 12, and we did not observe any facilitation from the left hand even when there was a nearby-hand effect under set size 8 .

The same trend of a right-hand facilitation was also observed in Cowan-Pashler's K. However, the interaction between hand position and set size in our $3 \times 2$ ANOVA failed to reach significance $(F=2.43, p=.10)$.
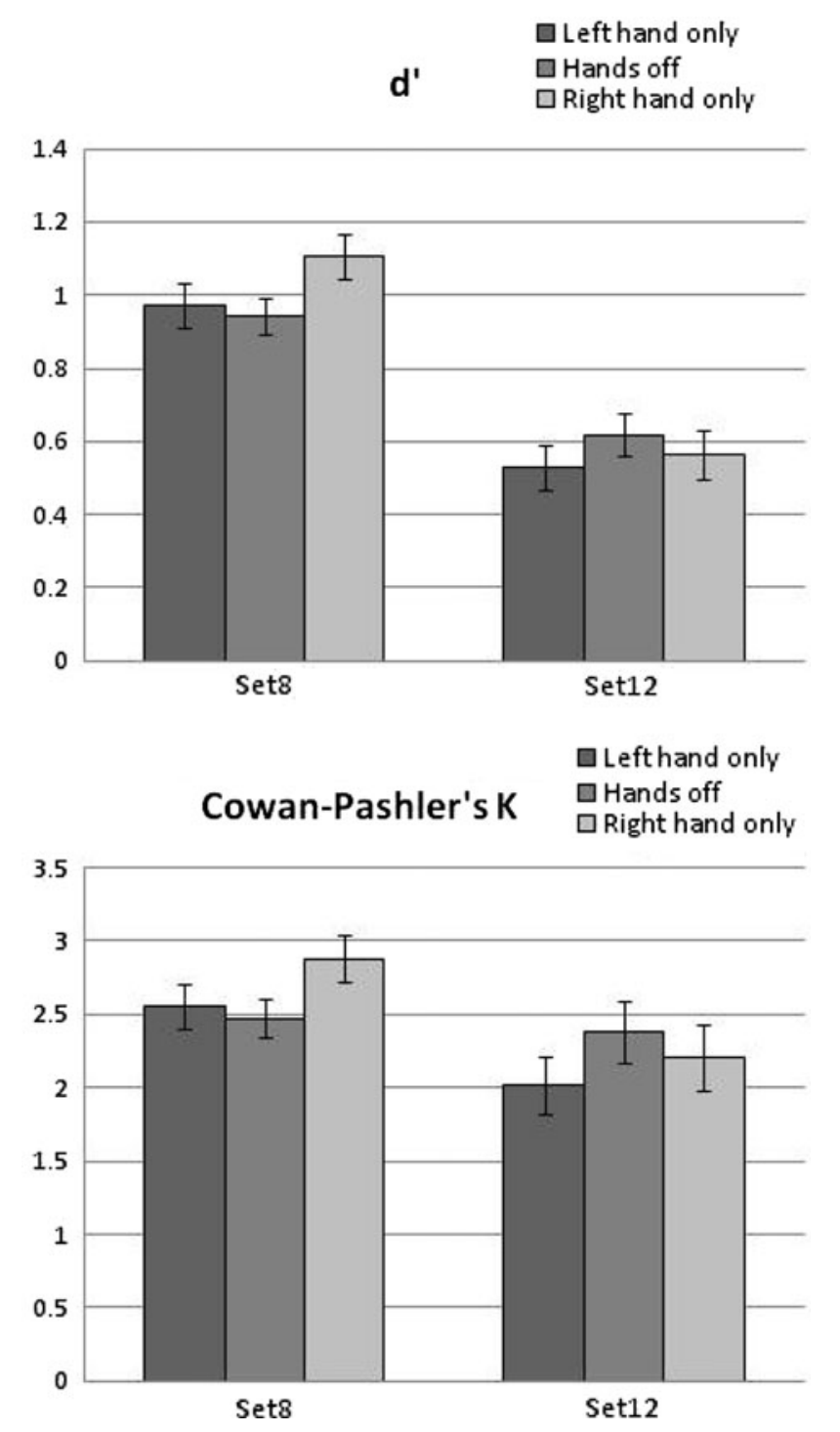

Fig. 5 Results from experiment 3. Error bars represent standard errors of the mean

\section{Regional analysis}

In the size 8 condition, we observed a significant facilitation from the right hand. Here, we again broke down the regional hit rate gains into their respective 6 columns (Fig. 6 , no vertical in this experiment) to see the distribution of the facilitatory effect. Similar to experiment 2, we aggregated the 6 columns into 3 in order to increase statistical power. However, one-way ANOVA did not yield any significant trend $(F=.017, \quad p=.98)$, which again indicates a uniformly distributed regional gain. There was also no hemifield difference (right hand: $\mathrm{t}=1.6, p=.22$; left hand: $\mathrm{t}=3.5, p=.07)$, which is consistent with our observation from experiment 2 under set size 8 . Although there was no significant facilitation from the hand under set size 12 , we performed the same regional analysis to see 
whether there was any noticeable pattern in hit rates. Interestingly, under set size 12 , the right hand produced a similar right-bias as in experiment 2 , at exactly half the magnitude (left: $93 \%$, right: 107\%; difference of $14 \%$ here and $28 \%$ in experiment $2 ; \mathrm{t}=7.13, p=.01$ ). No hemifield difference was found in the left-hand condition $(\mathrm{t}=.04, \quad p=.84)$. These results present a strikingly similar pattern to those from experiment 2 and suggest that perhaps the right hand is mainly responsible for the results of experiment 2. However, it is important to note that although we observed the same kind of uniform distribution and right-bias as in experiment 2 , the magnitude of the total regional gains under set size 8 here $(108 \%)$ was slightly lower than experiment $2(111 \%)$ and nonexistent in set size 12. Therefore, although the left hand itself is not sufficient to produce a nearby-hand facilitation, its addition to the right hand seems to be better than the right hand alone. This is discussed in more detail later and in the general discussion.

\section{Discussion}

The present experiment was designed to investigate the specific contribution and hit-rate distribution from each hand. By using a single-hand design, it is possible to compare the left- and right-hand effects with the bothhands setup (experiment 2). In short, we only observed hand facilitation under set size 8, but not under set size 12 . In addition, only the right hand boosted participants' performance, and the left hand was not effective. The hit gain from the right hand was uniformly distributed under set size 8 (Fig. 6 top row) and showed a right-bias under set size 12 (high task difficulty). These hit gain patterns are similar to the results of Experiment 2 and suggest that the right hand was the primary contributor in the previous experiments. This does not mean, however, that the left hand is not facilitatory. We now discuss each of these points separately.

\section{Nonlinear summation between hands}

The absence of a left-hand effect is quite surprising, but perhaps plausible with our right-handed participants. In fact, a recent study by Lloyd et al. (2010) also investigated the same nearby-hand effect with an attentional orienting paradigm and found that only the right hand would evoke an attentional prioritization in right-handed individuals. Thus, our findings here are consistent with their reports. Although this reasoning would imply that results of experiment 2 were entirely driven by the right hand alone, we do not think this is the case. Instead, the left hand may still be facilitatory, but only when the right hand is also present. This rationale is based on the fact that the overall hit rate gains dropped from $111 \%$ in experiment 2 (both
Fig. 6 Regional gains in hit rates from experiment 3 . Only the right-hand condition under set size 8 showed significant facilitation. The shape of the facilitation was uniformly distributed under size 8 , like experiment 2. However, when task became difficult (set size 12), the right bias re-appeared just like what we have observed in experiment 2
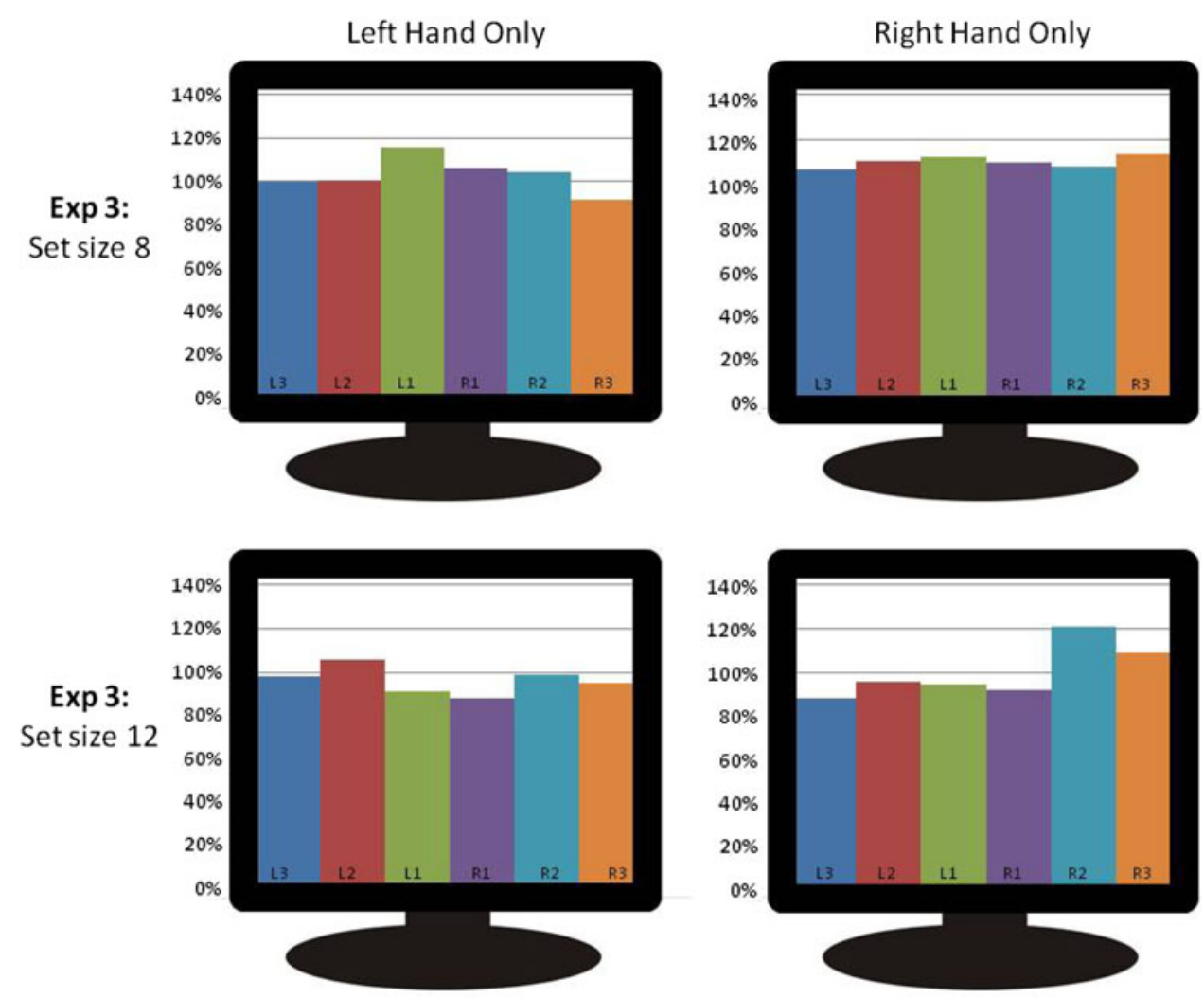
hands) to $108 \%$ in the present experiment (set size 8 right hand), and the reduced effect size (experiment $2: \eta^{2}=.6$; experiment 3 set size $8: \eta^{2}=.18$ ). Thus, it seems that the right hand alone could not fully replicate the experiment 2 results. Rather, there seems to be a nonlinear summation that occurs when both hands are nearby, and the effect weakens (right hand only) or disappears (left hand only) when only one hand is used.

The idea of nonlinear summation is not new. It emphasizes on interaction rather than sum, which is quite similar to the Gestalt principle "the whole is greater than the sum of the parts". Reports of nonlinear summation at the sensory level can be found in visual (Glantz 1971; Lee et al. 1989), auditory (Diesch and Luce 1997), and tactile (Ghazanfar and Nicolelis 1997) domains, where cellular or neural responses are greater when multiple receptive fields are stimulated simultaneously than the sum of individual responses. At the cognitive level, Franz and colleagues (Franz et al. 2000) have also demonstrated that the illusory effect of the Ebbinghaus Illusion is far greater, when the big and small central disks are placed side by side for a direct comparison between the two illusory percepts, than the sum of their individual effects from separate comparisons. Therefore, it is plausible that the induced attentional prioritization from both hands is nonlinearly stronger than the individual effect from each hand. It would be useful to see whether this nonlinear summation can also be observed in paradigms that use RT measures. To our knowledge, other studies investigating the nearby-hand effect have either used one hand (Lloyd et al. 2010; Reed et al. 2006, 2010) or both hands (Abrams et al. 2008; Davoli and Abrams 2009), but never both conditions together for direct comparison.

\section{No hand facilitation under set size 12}

Since the robust nearby-hand effect from experiment 2 was mainly driven by the nonlinear summation of both hands, the facilitatory effect was substantially weakened with only one hand in experiment 3. Therefore, it makes sense that the single-hand facilitation would be insufficient to improve performance when the task becomes increasingly difficult, such as the set size 12 condition in the present experiment. In addition, the Abrams et al. (2008) study suggested that when distracters were present (e.g., visual search in their study, change detection in our case), RT became longer when hands were placed near the display, suggesting an effect analogous to a speed-accuracy tradeoff that was brought forth by the attentional prioritization: deeper and more accurate processing of the stimuli that led to slower shift of attention. This implies that, when given insufficient time for a thorough processing of the stimuli, nearby-hand attentional prioritization can actually impair one's performance by prolonging visual processing time for each individual stimulus, which causes an incomplete encoding of the whole display when time runs out. In the case of change detection, it is possible that our participants were processing the colored squares at a slower speed and could not finish encoding all the squares by the time of stimuli offset. This would lead to less stored information in the VSTM and impair change detection performance, as we have observed in the present experiment. Ultimately, the cost outweighs the gain when too many stimuli were to be processed within a short timeframe. This perhaps would explain why the right hand was facilitatory only in the size 8 condition but not the size 12 condition. Interestingly, even when the nearby-hand effect was not strong enough, participants still exhibited a bias toward the right side. This suggests an attentional prioritization of the right side over the left and perhaps implies that our size 8 results could have also shown a right-bias if we did now allow sufficient display time for picture A and A'. Together, the present findings are consistent with previous reports and can be well explained by the notion of a nonlinear effect between the hands.

\section{General discussion}

In this paper, we have reported a superior change detection performance when one's right hand or both hands are near the changed objects. The right hand alone was able to improve performance, whereas the left hand could not. However, when both hands were near the display, there was a nonlinear boost of performance that surpassed the effect of the right hand. Regional analysis of hit gains showed that nearby right hand or both hands facilitated change detection uniformly across the entire display with a right-side prioritization.

The key difference that sets the present study apart from all previous investigations on the facilitatory effect of nearby-hand positions is the accuracy measures and the role of working memory in the change detection paradigm. As our results have indicated, nearby hands indeed enhance one's visual working memory and thereby improve change detection. We believe this facilitatory effect stems from the same underlying mechanism that previous studies have observed (Abrams et al. 2008; Cosman and Vecera 2010; Dufour and Touzalin 2008; Reed et al. 2006, 2010; Thura et al. 2008). Therefore, the benefits of nearby hands must have originated from an earlier stage of perception rather than acting directly on working memory. Previous studies have suggested a spatial prioritization of areas near the hands (Reed et al. 2006), but a slower shifts of attention from one item to the other (Abrams et al. 2008). The only interpretation to reconcile these findings with our accuracy 
data is a deeper focus of attention of objects near the hands, which allows one to detect rapid onset faster and encode them into visual working memory deeper, but at the cost of longer attentional shift when distracters are present due to the equally extensive visual analysis of all items.

Why should there be facilitatory attentional prioritization with the right hand, and even stronger with both hands, but not the left hand? We think this decreasing pattern of facilitation (from both hands to the right and then left) possibly reflects the frequency of interactions between each hand and the immediate environment in one's everyday life. That is, when interacting with objects, both the left and right hand are mostly seen working together in proximity (e.g., typing on a keyboard, asymmetrical uses such as opening a can of soda or washing dishes). This is especially true for most tasks that require focused attention. Therefore, placing both hands together may have over time become a cue, requesting attentional prioritization. In contrast, using only one hand occurs less often and is used mostly for easier tasks that requires less attention (e.g., holding a mug). Finally, using the left hand alone is almost never the first choice for the right-handed population. Thus, perhaps the most common hand position is the most facilitatory because it is indicative of the attentional demand of the forthcoming task. This would make an interesting prediction for the left-handed population as the left-handed individuals should have a reversed effect. Hand positions that are not indicative of demanding tasks can also be tested. An interesting study recently reported by Reed et al. (2010) showed that RT is more facilitated by nearby hand when stimuli are facing the palm instead of the back of the hand. This suggests that the visual system is sensitive not only to hand positions but also the functional space associated with that particular position. This would also explain our findings from the vertical-hands condition in experiment 2 , where the bottom hand was facing downward and not toward the screen because (1) the right hand was driving the facilitation when it was placed on top (facing the screen) and (2) when the right hand was placed at the bottom, its presence still made the left hand facilitatory (nonlinear summation), which allowed the left hand on top (facing the screen) to play a role in facilitating change detection. This line of research is definitely in its infancy, and the interaction between different hand positions and functional space, as well as the attentional prioritization they induce, remains to be explored by future research.

The neural correlate of the nearby-hand effect is still unclear at this point. Previous investigations have relied on the idea of bimodal neurons, which react to both visual and tactile signals, to explain the nearby-hand advantage. The strongest evidence comes from the Schendel and Robertson's study (2004), which suggested that arm positions near or within one's blind field can attenuate visual loss in patients with homonymous field deficits. The findings from this study, however, are still under rigorous debate. It has recently been shown that when patients' blind fields and other methodological details are properly controlled, the attenuation of visual loss due to nearby hands can no longer be observed (Smith et al. 2008). Thus, it remains unclear whether bimodal visual-tactile neurons are involved in the enhanced visual analysis we have reported here. Besides the bimodal neurons, one likely region to mediate the nearby-hand effect is the posterior parietal cortex, which is a part of the frontoparietal attentional network and marks the beginning of the dorsal "action" pathway (Mishkin et al. 1983). The posterior parietal cortex has been shown to update spatial mapping (Chao et al. in press; Merriam et al. 2003; Morris et al. 2007), direct attention (Rushworth and Taylor 2006), and most importantly, encoding and maintaining information in VSTM (Todd and Marois 2004; Tseng et al. 2010a; $\mathrm{Xu}$ and Chun 2006), all of which are necessary components of successful change detection. Therefore, the posterior parietal cortex's projection to the dorsal pathway and the attentional network makes it a likely region that mediates the nearby-hand effect.

In experiments 2 and 3, we observed a uniform distribution of facilitated hit rate. This uniform pattern is at odds with the graded RT facilitation reported by Reed et al. (2006). However, it is important to note that the orienting paradigm they used is very different from a change detection paradigm for two reasons. First, the presence of distractors in the change detection paradigm makes our task a discrimination task and not an onset detection task. Since the stimuli only stay on the screen for a brief period, the change detection paradigm encourages the observers to get a broad picture of the entire scene, rather than a detailed representation of only a few squares. Therefore, the uniform distribution we have observed here may be paradigmspecific as it is the optimal strategy in this context. Second, an orienting paradigm includes an exogenous cue, which effectively shifts attention away from the center. In contrast, the change detection task allows one to fixate at the center. This initial attention allocation is very useful in magnifying the RT differences but is not strategic in change detection where distractors are present, because attending initially to one side almost guarantees a lack of encoding on the opposite side. Thus, we think the differences between the graded and uniform facilitation between the two studies are perhaps context dependent and do not necessarily suggest inconsistent results between our study and Reed et al. (2006).

\section{Conclusion}

This study provides evidence for an extensive visual analysis of objects near the hands. When participants' right 
hand or both hands were on the display, change detection performance was more accurate and they held more items in their VSTM. Left hand alone was not enough to facilitate change detection, and the right hand seemed to drive most of the nearby-hand effect. When both hands were used, they together produced a stronger facilitation that could not be explained by the individual effect from the left and the right hand. Finally, when we break down the hit rates from different regions of the display according to their distances from the hands, the gains in hit rate were equal in magnitude across all regions, but with a prioritization for the right side.

Acknowledgments The author would like to thank Anya Levine, Benjamin Crow, James Billys, and Yaelang Jung for their help in conducting the experiments, and a special thanks to Anya Levine for her year-long dedication on this project. We also thank two anonymous reviewers for their helpful comments and the suggestion for experiment 3 .

Open Access This article is distributed under the terms of the Creative Commons Attribution Noncommercial License which permits any noncommercial use, distribution, and reproduction in any medium, provided the original author(s) and source are credited.

\section{References}

Abrams RA, Davoli CC, Du F, Knapp WK, Paull D (2008) Altered vision near the hands. Cognition 107:1035-1047

Bridgeman B, Peery S, Anand S (1997) Interaction of cognitive and sensorimotor maps of visual space. Percept Psychophys $59: 456-469$

Chao CM, Tseng P, Hsu TY, Su JH, Tzeng OJL, Hung DL, Muggleton NG, Juan CH. Predictability of saccadic behaviors is modified by transcranial magnetic stimulation over human posterior parietal cortex. Hum Brain Mapp (in press)

Cosman JD, Vecera SP (2010) Attention affects visual perceptual processing near the hand. Psychol Sci, doi:10.1177/095679761 0380697

Cowan N (2001) The magical number 4 in short-term memory: a reconsideration of mental storage capacity. Behav Brain Sci 24:1-185

Danckert J, Rossetti Y (2005) Blindsight in action: what can the different sub-types of blindsight tell us about the control of visually guided actions? Neurosci Biobehav R 29:1035-1046

Davoli CC, Abrams RA (2009) Reaching out with the imagination. Psychol Sci 20:293-295

Diesch E, Luce T (1997) Magnetic fields elicited by tones and vowel formants reveal tonotopy and nonlinear summation of cortical activation. Psychophysics 34:501-510

di Pellegrino G, Frassinetti F (2000) Direct evidence from parietal extinction of enhancement of visual attention near a visible hand. Curr Biol 10:1475-1477

Dufour A, Touzalin P (2008) Improved visual sensitivity in the perihand space. Exp Brain Res 190:91-98

Franz VH, Gegenfurtner KR, Bülthoff HH, Fahle M (2000) Grasping visual illusions: no evidence for a dissociation between perception and action. Psychol Sci 11:20-25
Ghazanfar AA, Nicolelis MAL (1997) Nonlinear processing of tactile information in the thalamocortical loop. J Neurophys 78:506-510

Glantz RM (1971) Visual adaptation: a case of nonlinear summation. Vis Res 12:103-109

Lee BB, Martin PR, Valberg A (1989) Nonlinear summation of Mand L-cone inputs to phasic retinal ganglion cells of the Macaque. J Neurosci 9:1433-1442

Lloyd DM, Azanon E, Poliakoff E (2010) Right hand presence modulates shifts of exogenous visuospatial attention in near perihand space. Brain Cogn 73:102-109

Luck SJ, Vogel EK (1997) The capacity of visual working memory for features and conjunctions. Nature 390:279-281

Merriam EP, Genovese CR, Colby CL (2003) Spatial updating in human parietal cortex. Neuron 39:361-373

Milner AD, Goodale MA (1995) The visual brain in action. Oxford University Press, New York

Mishkin M, Ungerleider LG, Macko KA (1983) Object vision and spatial vision: two cortical pathways. Trend Neurosci 6:414-417

Morris AP, Chambers CD, Mattingley JB (2007) Parietal stimulation destabilizes spatial updating across saccadic eye movements. Proc Natl Acad Sci USA 104:9069-9074

O'Regan JK, Deubel H, Clark JJ, Rensink RA (2000) Picture changes during blinks: Looking without seeing and seeing without looking. Vis Cogn 7:191-211

Pashler H (1988) Familiarity and visual change detection. Percept Psychophys 44:369-378

Perenin MT, Rossetti Y (1996) Grasping without form discrimination in a hemianopic field. Neuroreport 7:793-797

Posner MI, Walker JA, Friedrich FJ, Rafal RD (1987) How do the parietal lobes direct covert attention? Neuropsychologia 25:135-146

Reed CL, Grubb JD, Steele C (2006) Hands up: attentional prioritization of space near the hand. J Exp Psychol Hum 32:166-177

Reed CL, Betz R, Garza JP, Roberts RJ Jr (2010) Grab it! Biased attention in functional hand and tool space. Atten Percept Psychophys 72:236-245

Rushworth MF, Taylor PC (2006) TMS in the parietal cortex: updating representations for attention and action. Neuropsychologia 44:2700-2716

Schendel K, Robertson LC (2004) Reaching out to see: arm position can attenuate human visual loss. J Cogn Neurosci 16:935-943

Smith DT, Lane AR, Schenk T (2008) Arm position does not attenuate visual loss in patients with homonymous field deficits. Neuropsychologia 46:2320-2325

Thura D, Boussaoud D, Meunier M (2008) Hand position affects saccadic reaction times in monkeys and humans. J Neurophysiol 99:2194-2202

Todd JJ, Marois R (2004) Capacity limit of visual short-term memory in human posterior parietal cortex. Nature 428:751-754

Tseng P, Hsu TY, Muggleton NG, Tzeng OJL, Hung DL, Juan CH (2010a) Posterior parietal cortex mediates encoding and maintenance processes in change blindness. Neuropsychologia 48:1063-1070

Tseng P, Tuennermann J, Roker-Knight N, Winter D, Scharlau I, Bridgeman B (2010b) Enhancing implicit change detection through action. Perception 39:1311-1321

Vishton PM, Stephens NJ, Nelson LA, Morra SE, Brunick KL, Stevens JA (2007) Planning to reach for an object changes how the reacher perceives it. Psychol Sci 18:713-719

Wheeler ME, Treisman AM (2002) Binding in short-term visual memory. J Exp Psychol Gen 131:48-64

Xu Y, Chun MM (2006) Dissociable neural mechanisms supporting visual short-term memory for objects. Nature 440:91-95 